\section{Partograph utilization and associated factors among obstetric care providers in \\ North Shoa Zone, Central Ethiopia: a cross sectional study}

Negash Wakgari ${ }^{1}$, Abdella Amano ${ }^{2}$, Marta Berta ${ }^{2}$, Gizachew Assefa Tessema ${ }^{3}$

1. School of Nursing and Midwifery, College of Medicine and Health Sciences, Hawassa University, Hawassa, Ethiopia

2. Departments of Midwifery, College of Medicine and Health Sciences, University of Gondar, Gondar, Ethiopia

3. Department of Reproductive Health, Institute of Public Health, University of Gondar, Gondar, Ethiopia

\section{Abstract}

Background: Globally, prolonged and obstructed labor contributed to $8 \%$ of maternal deaths which can be reduced by proper utilization of partograph during labor.

Methods: An Institution based cross-sectional study was conducted in June, 2013 on 403 obstetric care providers. A pre-tested and structured questionnaire was used to collect data. Data was entered to EpiInfo version 3.5.1 statistical package and exported to SPSS version 20.0 for further analysis. Logistic regression analyses were used to see the association of different variables.

Results: Out of 403 obstetric care providers, $40.2 \%$ utilized partograph during labor.Those who were midwives by profession were about 8 times more likely to have a consistent utilization of the partograph than general practitioners ( $\mathrm{AOR}=8.13$, $95 \%$ CI: 2.67, 24.78). Similarly, getting on job training (AOR=2. 86, 95\% CI: 1.69, 4.86), being knowledgeable on partograph $(\mathrm{AOR}=3.79,95 \%$ CI: $205,7.03)$ and having favorable attitude towards partograph ( $\mathrm{AOR}=2.35,95 \%$ CI: 1.14, 4.87) were positively associated with partograph utilization. positively associated with partograph utilization.

Conclusion: Partograph utilization in labor monitoring was found to be low. Being a midwife by profession, on job training knowledge and attitude of obstetric care providers were factors affecting partograph utilization. Providing on job training for providers would improve partograph utilization.

Keywords: Ethiopia, obstetric care providers, partograph

DOI: http://dx.doi.org/10.4314/ahs.v15i2.30

$\begin{array}{ll}\text { Introduction } & \text { World Health Organization recommends the universal } \\ \text { Partograph is a } & \end{array}$ maternal and fetal condition plotted against time for intra-partum monitoring ${ }^{1,2}$. Its aim is to provide a pictorial overview of labor, to alert obstetric care providers about deviations in maternal, fetal condition and progress of labor ${ }^{3,4}$

\section{Corresponding author:}

Negash Wakgari

School of Nursing and Midwifery,

College of Medicine and Health Sciences,

Hawassa University, Hawassa, Ethiopia

Telephone: +2519-1709-3718 /

$+2519-1676-9260$

Fax number: +046-220-8755

Postal: 1560

Email: negashwakgari@yahoo.com
World Health Organization recommends the universal utilization of the partograph during labor ${ }^{5}$. Routine use of partograph is helpful to make better decisions for the diagnosis and management of prolonged and obstructed labor ${ }^{2,4}$

Globally, there were an estimated number of 289,000 maternal deaths in 2013. The sub-Saharan Africa region alone accounted for $62 \%(179,000)$ of maternal deaths followed by Southern Asia $(69,000)^{6}$. In spite of, government commitment in reduction of maternal death by three-quarters over the period of 1990 to 2015 , maternal mortality ratio is remained high, estimated at 676 per 100,000 live births in Ethiopia 7 . Obstructed labor accounted for $8 \%$ of maternal deaths. The reported incidence of obstructed labor expected to reach $20 \%$ in developing countries?. In Ethiopia obstructed or prolonged labor accounted for $13 \%$ of maternal deaths ${ }^{10}$. The women who experience obstructed labor usually suffer from postpartum hemorrhage, uterine rupture, puerperal sepsis and obstetric fistula $a^{11-13}$. Furthermore, it is highly associated with birth trauma, birth asphyxia, stillbirths, neonatal sepsis, and neonatal deaths. Besides, partograph is the best available tool but it is not widely used as it should be ${ }^{11}$. Thus; prevention of prolonged and obstructed labor by using partograph during labor is a key intervention in the reduction of maternal and perinatal morbidity and mortality ${ }^{14-16}$.

Data across African countries has shown that the utilization of partograph is poor despite preparing the tool that is simple and inexpensive for intra partum monitoring of labor 2,14,17-23 Similarly, different studies in Ethiopia revealed low utilization of the partograph ${ }^{13,19,24,25}$. The lack of preprinted partograph in the health institutions, being a general practitioner, poor knowledge and attitude towards partograph were reason for not using partograph during labor ${ }^{13,19,25}$; However, these all challenges to the use of the partograph can be resolved by provisions of pre-service and on job training on partograph ${ }^{13}$. Little is known about the partograph utilization and its associated factors; understanding this will help police makers, stakeholders, program planners and obstetric care providers to improve the quality of intrapartum care. Hence, this study provides information on the level of partograph utilization and its associated factors among obstetric care providers in North Shoa Zone, central Ethiopia.

\section{Methods}

\section{Study Setting}

The study was conducted from June 5 to 20, 2013 in North Shoa zone, central Ethiopia. North Shoa zone is one of the eleven zones in the Amhara national, regional state which contains 24 districts. Debre brehan, the city of the Zone is located at a distance of $130 \mathrm{~km}$ from Addis Ababa and at $795 \mathrm{~km}$ from the capital city of the region (Bahir Dar). With regard to health facilities there are 3 primary government hospital and 1 general hospital, 86 health centers, 387 health posts, one private hospital, 3 higher clinics, 7 medium clinics and 93 lower clinics in the zone. Among private institution only one hospital and one private clinic are providing delivery services. Regarding health professionals, there are about 354 clinical nurses, 121 midwives, 98 health officers, $45 \mathrm{BSc}$ nurses, 29 general practitioners and obstetrician working in the zone.
Study design

An institutional based cross-sectional study design was conducted to assess the level of partograph utilization and its associated factors among obstetric care providers in North Shoa zone, central Ethiopia.

\section{Population}

The source population included all obstetric care providers working in North Shoa zone. These included midwives, nurses, doctors and health officers working in both governmental and nongovernmental health institutions. The study participants were certified health personnel who provide care for the woman during labor and delivery and who consented to participate in the study. Obstetric care providers who took different leave (annual, sick and maternity leave) for more than five days during the data collection period were excluded from the study.

\section{Sampling methods}

The total number of health institutions in North Shoa zone is 581 (4 governmental hospitals, 1 private hospial, 86 health centers, 387 health posts and 103 private owned clinics). From these institutions, 4 hospitals, 86 health centers and 2 clinics (i.e., a total of 92 health institutions) are providing obstetric care services. The total numbers of health personnel who have been providing obstetric care in these 92 institutions are 457 (Data obtained from each instution by principal investigator via preliminary survey). All institutions providing delivery services in the zone were included in the study since the total number of obstetric care providers working in the study area was small.

The sample size was calculated using single population proportion formula, by considering the following assumptions. The proportion (p) of partograph utilization $=57.3 \%$ (13), 95\% confidence level of $\mathrm{Z} \mathrm{Z} \alpha / 2=$ $1.96,5 \%$ of absolute precision, and $5 \%$ non-response rate. Hence, the total sample size was 395 . Thirty one respondents were not available during the data collection period. Since the determined sample size and study population was almost equal, we included all (403) eligible obstetric care providers.

\section{Data collection instrumen}

A pre-tested and structured self-administered questionnaire was used for data collection. Different relevant literature was reviewed to develop the tool and to in- 
clude all the possible variables that address the objective before the actual work about the aim of the study, proof the study 10-14,26. The instrument was pretested on 30 cedures, and data collection techniques going through similar study participants who were working in other the questionnaires and clarification was given on ways health facilities. Findings from the pretest were used of collecting the data. to modify the instrument. The questionnaire was designed to obtain information on the professional char- Data analysis

acteristics of obstetric care providers, knowledge about The collected questionnaire was checked manually for partograph and their attitude towards partograph. its completeness, coded and entered into EpiInfo verKnowledge about partograph was measured by using sion 3.5.1 statistical package, then exported to SPSS vereight knowledge questions. In order to produce a more sion 20.0 for further analysis. Descriptive and summaobjective assessment of knowledge about partograph a ry statistics were done. Both bivariate and multivariate scoring method was devised and a knowledge score for logistic regression analysis was used to determine the each participant was obtained by adding up the score lossociation of each independent variable with the de for correct response given to selected questions in the pendent variable. Variables significant in bi-variate analquestionnaire. A score of mean value and above $\mathrm{e}^{-8}$ to ysis $(\mathrm{P}<0.2)$ were entered into a multivariate logistic knowledge related questions was considered as a good regression model to adjust the effects of cofounders o level of knowledge, while a score of less than mean the outcome variable. Odds ratio with their $95 \%$ confivalue (0-3) indicated poor level of knowledge $\mathrm{e}^{14}$. Provid- dence intervals were computed to identify the presence ers' attitude towards partograph utilization was assessed and strength of association, and statistical significance by using a 5-point Likert scale as individuals responding was declared if $\mathrm{p}<0.05$.

strongly agree for positive attitude was given scores of 5 and 1 for those who responded as strongly disagree, while the above scores was reversed for negative attitude questions.

Finally the total score was dichotomized into favorable and an unfavorable attitude taking the mean score as a cutoff point (Mean score or more $=$ favorable attitude and less than the mean score unfavorable attitude). Certified health personnel who provide care for the woman during labor and delivery were considered as an obstetric care provider. To measure the level of partograph utilization two steps had been under taken: The first step was, requesting the participants whether they have been using partograph or not (with Yes or No questions). Second step, the respondents who responded as "Yes" in the first step were required to answer" how often" they have been using partograph (occasionally, sometimes and routinely). Finally, obstetric care providers who have been using partographs routinely to monitor all laboring mothers were considered as partograph utilisers $2,4,5$ and while; those who were not using partographs, those who have been using sometimes and occasionally to monitor labor were considered as non atilized. Two BSc nurses facilitated the data collection process. Both facilitators were given one days training

Ethical considerations

Ethical approval was obtained from the institutional review board of the University of gondar and submitted to Zonal health department. Permission letter was granted from Zonal health department to respective health institutions. Written consent was obtained from each study subject prior to data collection process. Those respondents who were not willing to participate in the study were not forced to be involved. They were also informed that all data obtained from them would be kept confidential by using codes instead of any personal identifiers and is meant only for the purpose of he study.

\section{Results}

Socio-demographic characteristics of study participants

A total of 403 obstetric care providers were included in the study (response rate $=94.5 \%$ ). About half $(50.4 \%)$ of obstetric care providers were males. The mean age of the respondents were 26.2 years (Standard deviation $(\mathrm{SD})=4.26)$. Most $(80.4 \%)$ of them were working at the health center. Regarding their profession $(45.4 \%)$ were Nurses. Two hundred thirty five $(58.3 \%)$ had a diploma. The duration of practice ranged between 1 and 37 years $(\mathrm{SD}=4.44)$ (Table1).

Table1: Socio-demographic characteristics of obstetric care providers in North Shoa Zone, central Ethiopia, 2013(n=403).

\begin{tabular}{lll}
\hline Variables & Frequency & Percent \\
\hline Sex & & \\
Male & 203 & 50.4 \\
Female & 200 & 49.6 \\
Age in years & & \\
$20-24$ & 184 & 45.7 \\
$25-29$ & 180 & 44.7 \\
30 or more & 39 & 9.6 \\
Health facilities & & \\
Health center & 324 & 80.4 \\
Hospital & 75 & 18.6 \\
Private facilities & 4 & 1 \\
Profession & & \\
Nurse & 183 & 45.4 \\
Midwifery & 111 & 27.6 \\
Health officer & 88 & 21.8 \\
$\begin{array}{l}\text { General practitioner } \\
\text { (MD) }\end{array}$ & 21 & 5.2 \\
Qualification level & & \\
Diploma & 235 & 58.3 \\
Bsc & 140 & 34.8 \\
General practitioner & 21 & 5.2 \\
MSc & 7 & 1.7 \\
Year of experience & & \\
(in years) & & \\
1-5 & 237 & 58.8 \\
6-10 & 140 & 34.8 \\
11 or more & 26 & 6.4 \\
\hline & & \\
\hline
\end{tabular}

\section{Partograph utilization}

The majority $327(81.1 \%)$ of obstetric care provider reportedly utilizes partograph to monitor labor. O those who were utilising partograph $162(40.2 \%)$ used routinely for all laboring mothers.

\section{Reasons for not utilizing partograph}

Among reasons cited by respondents for not utilising partograph to monitor labor; using different monitoring tools accounted for $(52.6 \%)$. Similarly, among those who were using the partograph sometimes or occasionally; $97(58.79 \%)$ of them were using different monitoring tools like clinical records, monitoring charts, piece of papers to monitor labor other than partograph, while $68(41.21 \%)$ of them were citing shortage of staff as barrier for not utilizing partograph routinely during labor (Figure 1). 
Figure 1: Reasons of obstetric care providers for not practicing partograph, North Shoa, Northern Ethiopia, 2013(n=76).

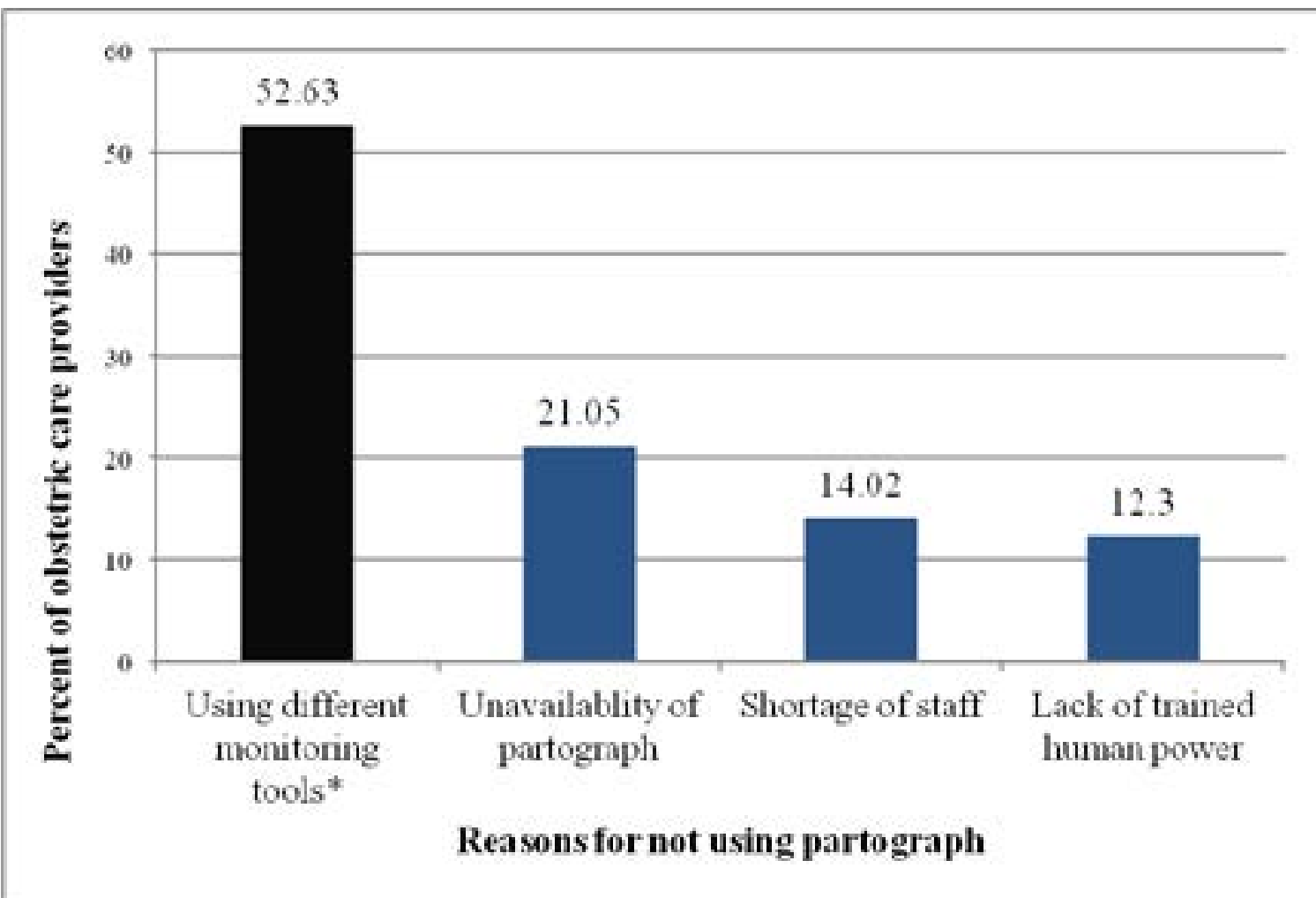

*Using different monitoring tools- Clinical records, monitoring charts, piece of papers

Knowledge and attitude of obstetric care providers towards partograph utilization

multiple logistic regression analysis: profession, on job training, knowledge and attitude of obstetric care proRegarding the knowledge about partograph, more than viders towards partograph were significantly associated two thirds $(70.5 \%)$ of the care providers were knowl- with partograph utilization. Those who were midwives edgeable. Almost all $(99.8 \%)$ of the participants were by profession were about 8 times more likely to have learned about partograph while at college or university. a consistent utilization of the partograph than general More than half $(59.3 \%)$ of them received on the job practitioners (AOR $=8.13,95 \%$ CI: 2.67, 24.78). Those training on partograph. The majority $(83.6 \%)$ of ob- obstetric care providers who received on the job trainstetric care providers had a favorable attitude towards ing on partograph were about 3 times more likely to utipartograph.

Factors associated with partograph utilization In bivariate analysis the factors found to be significantly associated with partograph utilization were: health facility they were working, profession, qualification stetric care providers towards partograph. However, in lize partograph than who haven't received on- job training $(\mathrm{AOR}=2.86,95 \% \mathrm{CI}: 1.69,4.86)$. In addition, those who were knowledgeable on partograph were about 4 times more likely to utilize partograph than the non knowledgeable (AOR $=3.79,95 \%$ CI: 2.05, 7.03). Furthermore, those who had a favorable attitude towards partograph utilization were about 2 times more likely to utilize partograph than those with non favorable attitude $(\mathrm{AOR}=2.35,95 \% \mathrm{CI}: 1.14,4.87)$ (Table 2$)$.
Table 2: Bivariate and Multivariate analyses of factors associated with partograph utilization among obstetric care providers, central Ethiopia, July, 2013(n=403).

\begin{tabular}{|c|c|c|c|c|c|}
\hline \multirow{2}{*}{ Variables } & \multicolumn{2}{|c|}{ Partograph Utilization } & \multicolumn{2}{|c|}{ OR $(95 \% \mathrm{CI})$} & \multirow[b]{2}{*}{ OR(95\% CI) } \\
\hline & Utilized & Not utilized & COR $(95 \% \mathrm{CI})$ & & \\
\hline \multicolumn{6}{|l|}{ Health facilities } \\
\hline Hospitals & $37(47.4 \%)$ & $41(52.6 \%)$ & $1.44(0.88,2.37)$ & & \\
\hline Health centers & $125(38.5 \%)$ & $200(61.5 \%)$ & 1 & & \\
\hline \multicolumn{6}{|l|}{ Profession } \\
\hline Midwifery & $81(73.0 \%)$ & $30(27.0 \%)$ & $6.75(2.39,19.01)$ & & $8.13(2.67,24.78)^{*}$ \\
\hline Nurse & $58(31.7 \%)$ & $125(68.3 \%)$ & $1.16(0.43,3.14)$ & & $1.1(0.37,3.11)$ \\
\hline Health officer & $17(19.3 \%)$ & $71(80.7 \%)$ & $0.59(0.20,1.77)$ & & $0.41(0.13,1.28)$ \\
\hline General practitioner & $6(28.6 \%)$ & $15(71.4 \%)$ & 1 & & \\
\hline Qualifications & & & & " & \\
\hline Diploma & $112(47.7 \%)$ & $123(52.3 \%)$ & 1 & & \\
\hline BSc and above & $50(29.8 \%)$ & $118(70.2 \%)$ & $0.46(0.31,0.71)$ & & \\
\hline \multicolumn{6}{|l|}{ On-job training } \\
\hline Yes & $122(51.0 \%)$ & $117(49.0 \%)$ & $3.23(2.09,5.01)$ & & $2.86(1.69,4.86) *$ \\
\hline No & $40(24.4 \%)$ & $124(75.6)$ & 1 & 1 & \\
\hline \multicolumn{6}{|l|}{ Knowledge } \\
\hline Knowledgeable & $138(48.6 \%)$ & $146(51.4 \%)$ & $3.74(2.26,6.19)$ & & $3.79(2.05,7.03) *$ \\
\hline Not Knowledgeable & $24(20.2 \%)$ & $95(79.8 \%)$ & 1 & 1 & \\
\hline \multicolumn{6}{|l|}{ Attitude } \\
\hline Favorable & $145(43.0 \%)$ & $192(57.0 \%)$ & $2.18(1.20,3.94)$ & & $2.35(1.14,4.87) *$ \\
\hline Unfavorable & $17(25.8 \%)$ & $49(74.2 \%)$ & 1 & 1 & \\
\hline
\end{tabular}

Discussion

reason for this gap, as such, they may not have had adeThis study attempted to identify level of partograph quate knowledge about partograph as compared to othutilization and associated factors among obstetric care er professions ${ }^{17}$. In the studies of the Niger Delta $(\mathrm{Ni}$ providers in North Shoa Zone, Central Ethiopia. The geria), Gambia and South Africa; the participants were level of partograph utilization was found to be $40.2 \%$ only midwives by profession with a great chance to be (95\% CI: 35.2, 45.2). This finding is lower than the stud- trained on partograph than other professions which ies of Addis Ababa $(57 \%)^{13}$, Benin $(98 \%)^{27}$, the Niger might in turn improves their knowledge and skill of its delta of Nigeria $(98.8 \%)$ and $(50.6 \%)^{2}$, Gambia $(78 \%)^{29}$ utilization. In contrast to this, relatively large numbers and South Africa $(64 \%)^{28}$. However, it was higher than of study participants from different professions particthe findings of Ethiopian hospitals $(13 \%)^{25}$, the Am- ipated in our study.

hara region $(29 \%)^{19}$, Uganda $(30 \%)^{22}$, Nigeria $(8.4 \%)$,

$(32.3 \%)$ and $(9.8 \%)^{14,17,18}$. These differences might be In the present study, the reasons for not using pardue to differences in the place of the study that may be tograph during labor were using monitoring tools othe explained with different strategies in partograph imple- than partograph, lack of trained human power, shortmentation, different levels of knowledge and attitudes age of staff, and un-availability of the partograph. This of care providers towards partograph utilization. age of staff, and un-availability of the partograph. This
finding is consistent with the studies in Nigeria and South Africa ${ }^{2,18,28}$.

The other possible explanation could be the difference The profession of the obstetric care provider is one of in the data collection procedure, sample size and time the factors for partograph utilization. Partograph utiligap since, as time goes on, there is a change in policy, zation was higher when the providers were midwives strategy and improvement in implementation of the than general practitioners. This agrees with a study conpartograph. Involvement of community health exten- ducted in nineteen Ethiopian hospitals ${ }^{25}$. This might sion workers in the study of Nigeria may also another be due to the fact that midwife obstetric care providers 
had more of chance of being assigned in delivery wards and consequently received training on partograph utilization which might in turn have improved their knowledge and skills to utilize partograph than others. This concept is in agreement with the study done in Amhara region ${ }^{19}$ in which midwives had better knowledge about partograph. Secondly, as obstetric care is their major duty; they might have a better understanding about partograph utilization than others.

On-job training on partograph had a significant association with partograph utilization. Obstetric care providers who received on the job training on partograp were about 3 times more likely to utilize partograph than those who had not received on- job training. This might be due to the fact that, obstetric care providers who received on-job training had better knowledge about partograph than others ${ }^{13,14,19}$; that in turn improves their partograph utilization.

Those providers who were knowledgeable about partograph were about 4 times more likely to utilize partograph than the non knowledgeable. This is in line with the study done in the Niger delta region of Nigeria ${ }^{2}$. This implies that, having knowledge about partograph is important to implement partograph during labor. Furthermore, the study found that attitude as anothe factor influencing partograph utilization. Partograph utilization was significantly higher among obstetric care providers who had a favorable attitude as compared to those who had the unfavorable attitude. This could be due to the fact that, having a good attitude towards partograph utilization might come after having knowledge about partograph that may influence the utilization of partograph.

\section{Limitations}

Since information about partograph utilization was obtained from respondents through self administered questionnaires, rather than observation; response bias and social desirability bias are the potential limitations of this study. However, numerous scientific procedures were employed to minimize the possible effects. To reduce the response bias, for instance, the aim of the study was discussed with respondents in order to obtain genuine response. In addition, procedures such as supervision, pretest of data collection tool, and adequate training of data collectors and supervisors were utilized.
USA:Central Statistical Agency and ICF International;2013.

8. Nour MN. Women's health in the developing world An Introduction to Maternal Mortality. Rev Obstet Gynecol 2008;1(2):77-81

9. World Health Organization. The World Health Report 2005: Make every mother and Child count. Geneva: World Health Organization. 2005

10. Federal Democratic Republic of Ethiopia Ministry of Health. Maternal Death Surveillance and Response (MDSR) Technical Guideline, Addis Ababa, Ethiopia,2012.

11. Magon N. Partograph Revisited. Int I Clin Cases Investig 2011; 3(1):1-6.

12. Kalembo FW, Zgambo M. Public Obstetric Fistula: A hidden public health problem Sub -Saharan Africa. ASSJ 2012;41:1-8.

13. Yisma E, Dessalegn B, Astatkie A ,Fesseha N. Knowledge and utilization of partograph among obstetric care givers in public health institutions of $\mathrm{Ad}-$ dis Ababa, Ethiopia. BMC pregnancy and cbildbirth 2013;13(17):1-9.

14. Fawole AO, Hunyinbo KI, Adekanle DA. Knowledge and utilization of the partograph among obstetric care givers in South West Nigeria. Afr J Reprod Health 2008;12(1):1-29.

15. Qureshi ZP, Sekadde-Kigondu C, Mutiso SM Rapid assessment of partograph utilisation in selected maternity units in Kenya. East Afr Med J 2010;87(6):235241.

16. Ganesh D. Preventing prolonged labor by using partograph. Int J Gynecol Obstet 2008; 8(2):1.

17. Oladapo OT, Daniel OJ, Olatunji AO. Knowledge and use of the partograph among healthcare personnel at the peripheral maternity centres in Nigeria. $\mathrm{OOb}$ stet Gynaecol 2006; 26(6):538-541.

18. Fawole AO, Adekanle DA, Hunyinbo KI. Utilization of the partograph in primary health care facilities in Southwestern Nigeria. Niger J Clin Pract 2010;13(2):200204. BAR 2012;3(5):391-396

5. WHO. World Health Organization partograph in management of labor. Lancet 1994;343:1399-1404.

6. WHO, UNFPA, UNICEF, UN, World Bank. Trend in Maternal mortality: 1990 to 2013: Estimates Developed by WHO,UNICEF,UNFPA, The World Bank and the United Nations Population Divisions. Geneva:World Health Organization;2014.

7. Central Statistical Agency (Ethiopia) and ICF International: Ethiopia Demographic and Health Survey 2011:Addis Ababa. Ethiopia and Calverton, Maryland,

19. A A ment of knowledge and utilization of the partograph mong health professionals in Amhara region, Ethiopia. SJCM 2013;2(2):26-42

20. Nyamtema AS, Urassa DP, Massawe S, Massawe A, Lindmark G, Van Roosmalen J. Partogram use in the Dar es Salaam perinatal care study. Int J Gynecol Obstet 2008; 100:37-40

21. Fawole AO, Fadare O. Audit of use of the partograph at the University College Hospital, Ibadan. Afr Med Sci 2007;36(3):273-278

22. Ogwang S, Karyabakabo Z, Rutebemberwa E. Assessement of partograph use during labour in Rujumbura Health Sub District, Rukungiri District, Uganda. Afr Health Sci 2009; 9(1):27-34.

23. Ghebrehiwet M, Morrow Richard H. Availablity,utilization and Quality of normal delivery and emergency obstetric care in Eritrea. Jema,17-21.

24. Yisma E, Dessaleon B, Astatkie A, Fesseha N. Completion of the modified World Health Organization (WHO) partograph during labour in public health institutions of Addis Ababa, Ethiopia. Reproductive Health 2013;10(23):1-7.

25. Getachew A, Ricca J, Cantor D, Rawlins B, Rosen $\mathrm{H}$, Tekleberhan A, et al. Quality of care for prevention and management of common maternal and newborn complications:A study of Ethiopia's Hospitals. MCHIP, 2011,21231-3492.

26. Maternal Health Task Force. The Partograph: A simple tool that is quite complicated. http://maternalhealthtaskforce.org/2011.Accessedon Dec.5,2013. 27. Azandegbe N, Testa J, Makoutode M. Assessment of partogram utilization in Benin. Sante 2004;14:251-

28. Mathibe-Neke JM, Lebeko FL, Motupa B. The partograph: A labor management tool or a Midwifery record?. Int. J. Nurs. Midwifery 2013;5(8):145-153.

29. Burama B, Kao HC, Gua LM, Lin CK. Partograph use among Midwives in the Gambia. AJM 2013; 